\title{
Mesomorphism, molecular structure and dynamics of polydiethylsiloxane*
}

\author{
Gerhard Kögler and Kyriakos Loufakis \\ Institut für Makromolekulare Chemie, Hermann-Staudinger-Haus, \\ Stefan Meier Strasse 31, D-7800 Freiburg, FRG \\ and Martin Möller† \\ Chemische Technologie, Twente University, PO Box 217, Enschede 7500 AE, \\ Netherlands
}

(Received 23 May 1989; revised 20 November 1989; accepted 1 February 1990)

\begin{abstract}
The correlation between the phase behaviour of polydiethylsiloxane (PDES) and conformational and motional changes at the various disordering transitions has been investigated by nuclear magnetic resonance (n.m.r.), dielectric relaxation and shear experiments. Diffusive rotation of the chain segments around the long axis of the molecules is indicated by ${ }^{29} \mathrm{Si}$ n.m.r. below the isotropization transition. The remarkably fluid character is explained in part by the coexistence of anisotropic and isotropic motional states of the $-\mathrm{OSiEt}_{2}-$ segments, which indicate microscopic domain formation. The molecular motion in the $\alpha_{2}-$ and $\beta_{2}$-phases is restricted but still fast with respect to the ${ }^{29} \mathrm{Si}$ n.m.r. timescale. Transmission electron micrographs show, besides chain-folded lamellae, also extended-chain lamellae. These differences in the morphologies can explain why the interconversion of $\alpha_{2}$-PDES into the thermodynamically more stable $\beta_{2}$-polymorph is slow, in spite of the pronounced mobility of the polymer segments. Long-range reorganization processes have to be considered to allow the morphological changes observed by electron microscopy.
\end{abstract}

(Keywords: mesomorphism; molecular structure; dynamics; polydiethylsiloxane)

\section{INTRODUCTION}

High-molecular-weight polydiethylsiloxane exists in two crystalline polymorphs, $\alpha$ - and $\beta$-PDES. Each of them undergoes a disordering transition from $\alpha_{1}$ to $\alpha_{2}$ and from $\beta_{1}$ to $\beta_{2}$ respectively ${ }^{1-13}$. Annealing experiments allowed us to identify the $\beta$-modification as the thermodynamically stable one ${ }^{8}$. Both polymorphs convert prior to isotropization into a phase $\mu$, which is also referred to as the $\alpha_{\mathrm{m}}$-phase $\mathrm{p}^{3,4}$. The observed fractions of $\alpha$ - or $\beta$-polymorph depend on the rate at which the sample is cooled from the $\mu$-phase. Increasing cooling rates result in increasing amounts of the $\alpha$-modification. The $\alpha_{2}-\mu$ transition occurs at $280 \mathrm{~K}$, the $\beta_{2}-\mu$ transition at $290 \mathrm{~K}$. The $\mu$-phase melts in a rather broad temperature range between about 300 and $320 \mathrm{~K}$. The change in enthalpy at the isotropization transition is small compared to that of the $\alpha_{1}-\alpha_{2}$ and $\alpha_{2}-\mu$ as well as the $\beta_{1}-\beta_{2}$ and $\beta_{2}-\mu$ transitions. It contributes only about $3 \%$ to the total entropy of fusion, which differs little for the $\alpha$ - and the $\beta$-polymorph ${ }^{4,8}$. The formation of the $\mu$-phase has not been observed for low-molecular-weight fractions of PDES, which contained large amounts of cyclics ${ }^{9,10}$. N.m.r. ${ }^{8,11-13}$ and Raman experiments ${ }^{7}$ showed the onset of molecular motion at the various solid-solid transitions, which results in dynamic conformational disordering. In the present paper, we discuss morphological differences and solid-state properties of $\alpha_{2^{-}}, \beta_{2^{-}}$and $\mu$-PDES in relation to the molecular structure and dynamics.

* Dedicated to Professor Dr H. Sinn on the occasion of his 60th birthday

† To whom correspondence should be addressed

0032-3861/90/081538-08

(C) 1990 Butterworth-Heinemann Ltd

1538

\section{EXPERIMENTAL}

\section{Materials}

Sample preparation and characterization (gel permeation chromatography, osmosis and thermal analysis) have been described before ${ }^{8}$. The investigated sample had a number-average molecular weight of 93000 as obtained from osmotic pressure experiments. Gel permeation chromatography according to a calibration by standard polystyrenes gave molecular weights of $M_{\mathrm{n}}=50000$ and $M_{\mathrm{w}}=83000$.

The pure $\beta$-polymorph was prepared by several cooling and heating cycles between 265 and $278 \mathrm{~K}$, where the cooling rate was $1 \mathrm{~K} \mathrm{~min}^{-1}$. The sample with the highest fraction of the $\alpha$-modification was prepared by quenching the sample from the mesophase using a liquid butane/ propane mixture, cooled with liquid nitrogen.

\section{Methods}

D.s.c. measurements were performed on a PerkinElmer DSC 7. The instrument was calibrated with high-purity samples of indium, gallium and cyclohexane. The transition temperatures were recorded with a heating rate of $10 \mathrm{~K} \mathrm{~min}^{-1}$. Typically, the sample weight was chosen between 0.5 and $1 \mathrm{mg}$. Temperature accuracy was better than $\pm 1 \mathrm{~K}$. The onset of the recorded endotherm on heating is given for the transition temperatures. Transition entropies were calculated assuming equilibrium, i.e. $T_{\mathrm{tr}} \Delta S_{\mathrm{tr}}=\Delta H_{\mathrm{tr}}$.

Solid state ${ }^{29} \mathrm{Si}$ and ${ }^{13} \mathrm{C}$ n.m.r. spectra were recorded on a Bruker CXP 300 spectrometer at $59.63 \mathrm{MHz}$ and $75.47 \mathrm{MHz}$ respectively. A Bruker double-bearing magic- 
angle spinning (MAS) probe, which was modified for variable-temperature experiments, was used. Two different pulse sequences were used to generate the spectra. The usual spin-lock cross-polarization (CP) sequence ${ }^{14}$ was applied for the spectra in the low- and the hightemperature phase. In this case, a $90^{\circ}$ pulse $(3.9 \mu$ s for ${ }^{29} \mathrm{Si}$ ) was followed by a mixing time of $1-4 \mathrm{~ms}$ and an acquisition time of $30-150 \mathrm{~ms}$. The spectra of the mesophase and of the melt were obtained without cross-polarization, a simple $90^{\circ}$ pulse in combination with high-power proton decoupling being applied. Delay times between 10 and $600 \mathrm{~s}$ were chosen, to be at least five times as long as $T_{1}$ to ensure complete relaxation between each pulse experiment. Temperatures were measured by means of a thermocouple adjacent to the rotor with an accuracy of $\pm 3 K$.

Spin-lattice relaxation times of PDES in the $\mu$-phase and in the melt were measured by means of an inversion recovery pulse sequence. A $180^{\circ}$ pulse was followed by a variable delay time before acquisition. To determine $T_{1}$ of $\alpha$ - and $\beta$-PDES, an additional $90^{\circ}$ pulse was employed after the cross-polarization sequence, which resulted in a $-z$ orientation of the magnetization. After a variable delay for relaxation, a simple $90^{\circ}$ pulse was employed to allow acquisition.

Electron micrographs were obtained on a Zeiss EM 902 with an acceleration voltage of $80 \mathrm{kV}$. One-stage carbon replicas were prepared from freshly fractured surfaces at $170 \mathrm{~K}$ with a 'Gefrierätzanlage Balzer BAF'. The specimens were shaded with platinum under rotation at an angle of $30^{\circ}$ or without rotation under an angle of $45^{\circ}$.

Dielectric relaxation was measured with a HewlettPackard Impedanz Analysator HP 4192 with a frequency of $10000 \mathrm{~Hz}$. Two gold-coated quartz plates with a spacer of $75 \mu \mathrm{m}$ width were used as condenser plates.

Birefringence micrographs were obtained by means of a polarization microscope (Leitz Ortholux II Pol BK) with a hot stage (Mettler FP52).

Creep recovery experiments were carried out with a Rheometrics Stress Rheometer, RSR. A $25 \mathrm{~mm}$ cone-andplate fixture with a cone angle of 0.1 rad was used. A constant torque was employed long enough to observe constant shear rates. Extrapolation of the steady shear viscosities thus obtained yielded the $\eta_{0}$ values. Temperature control was achieved by means of a thermostated liquid bath with a constancy of $\pm 0.5^{\circ} \mathrm{C}$.

\section{RESULTS}

Recently we reported solid-state ${ }^{13} \mathrm{C}$ and ${ }^{29} \mathrm{Si} \mathrm{n.m.r.} \mathrm{data}$ on PDES and discussed them in comparison with the thermal behaviour ${ }^{8}$. Changes of the isotropic ${ }^{29} \mathrm{Si}$ resonance at the solid-solid transitions $\left(\alpha_{1}-\alpha_{2}, \beta_{1}-\beta_{2}\right.$, $\alpha_{2}-\mu$ and $\beta_{2}-\mu$ ) indicate dynamic disordering of the conformation of the polymer backbone. The two polymorphs $\alpha_{1}$ and $\beta_{1}$ can be clearly distinguished by their isotropic ${ }^{29} \mathrm{Si}$ and ${ }^{13} \mathrm{C}$ resonances. A considerable upfield shift of the isotropic ${ }^{29} \mathrm{Si}$ resonance line demonstrates a change of the polymer backbone conformation. Only small differences are observed for $\alpha_{2}$ - and $\beta_{2}$-PDES in the isotropic ${ }^{29} \mathrm{Si} \mathrm{n.m.r.} \mathrm{chemical} \mathrm{shifts,} \mathrm{and} \mathrm{the} \mathrm{two}$ modifications become indistinguishable already $50 \mathrm{~K}$ below the transition to the $\mu$-phase. The corresponding difference in the ${ }^{13} \mathrm{C}$ spectra is slightly larger and can be observed even $20 \mathrm{~K}$ below the upper disordering transition. The results demonstrate nearly identical conformations of the two polymorphs in their hightemperature phase. Liquid-like disordering is indicated for the $\mu$-phase by the ${ }^{13} \mathrm{C}$ and ${ }^{29} \mathrm{Si}$ chemical shifts, which are the same as those of the isotropic melt.

Variations in the motional state of PDES segments are also indicated by spin-lattice relaxation experiments. The $T_{1}$ times showed significant changes, which are consistent with the onset of different motional processes discussed above. Corresponding to the $\beta_{1}-\beta_{2}$ transition, ${ }^{29} \mathrm{Si} T_{1}$ drops sharply from $200 \mathrm{~s}$ at $180 \mathrm{~K}$ and $270 \mathrm{~s}$ to $30 \mathrm{~s}$ at $220 \mathrm{~K}$. Within the $\beta_{2}$-state, $T_{1}$ increases to $52 \mathrm{~s}$ at $250 \mathrm{~K}$ and $80 \mathrm{~s}$ at $270 \mathrm{~K}$. An additional significant decrease of the $T_{1}$ time is observed at the transition to the $\mu$-phase: $T_{1}$ drops to $25 \mathrm{~s}$ at $300 \mathrm{~K}, 20 \mathrm{~s}$ at $315 \mathrm{~K}$ and $23 \mathrm{~s}$ at $330 \mathrm{~K}$. Upon isotropization, no further change of the $T_{1}$ times is observed within the experimental error.

The results are in agreement with $\operatorname{Raman}^{7}$ and ${ }^{1} \mathrm{H}$ n.m.r. ${ }^{11,12}$ experiments, which indicated the onset of side-chain motion and low-amplitude - $\mathrm{Si}-\mathrm{O}$ - segment torsion at the lower disordering transition. Litvinov et al. gave a detailed picture of the segmental motions and the corresponding frequencies based on their very careful ${ }^{1} \mathrm{H}$ n.m.r. second moment and relaxation experiments ${ }^{11,12}$. This is the onset of side-chain mobility and torsional vibrations of the $\mathrm{Si} \mathrm{O}$ links upon the lower disordering transition and polymer backbone reorientation above the second disordering transition.

\section{Nuclear magnetic resonance}

The motional states of the PDES molecules can be observed directly by the ${ }^{29} \mathrm{Si}$ chemical-shift anisotropies $(C S A)$. This is shown in Figure 1 for $\beta$-PDES. No differences could be observed on recording the CSA of a sample that consisted mainly of $\alpha$-PDES. The ${ }^{29}$ Si $C S A$ is not significantly motionally narrowed below the lower solid transition. The spectrum is almost axially symmetric, with $v_{x}-v_{z}=3.1 \mathrm{kHz}$. In contrast, motional narrowing is observed for the ${ }^{13} \mathrm{C} \mathrm{CSA}$ already below the lower disordering transition. The total linewidth for the $\mathrm{CH}_{3}$ and the $\mathrm{CH}_{2}$ carbons changed from about $1.7 \mathrm{kHz}$ at $100 \mathrm{~K}$ to $1.1 \mathrm{kHz}$ at $190 \mathrm{~K}^{8}$. Thus, side-chain mobility is observed below the lower disordering transition, while the polymer backbone remains fixed on the n.m.r. timescale.

The ${ }^{29} \mathrm{Si} C S A$ becomes slightly narrowed and remains almost axially symmetric when the temperature is raised above the lower transition, $v_{x}-v_{z}=2.9 \mathrm{kHz}$. This can be explained by the onset of small-amplitude $-\mathrm{Si}-\mathrm{O}-$ backbone motions. The deviation from axial symmetry becomes more pronounced with increasing temperature. A non-axially symmetric spectrum with $\eta=0.14$ and $v_{x}-v_{z}=2.6 \mathrm{kHz}$ was recorded at $280 \mathrm{~K}$. This indicates increasing amplitudes of the molecular motion. No significant difference between the spectra of $\beta_{2^{-}}$and $\alpha_{2}$-PDES could be observed.

Additional narrowing of the ${ }^{29} \mathrm{Si} C S A$ from $2.6 \mathrm{kHz}$ to $1.1 \mathrm{kHz}$ at the transitions to the $\mu$-phase reveals the onset of large-amplitude molecular mobility of the backbone. The spectrum at $293 \mathrm{~K}$ is again axially symmetric with $v_{x}-v_{z}=1.1 \mathrm{kHz}$ and $\eta=0$. The axial symmetry indicates diffusive averaging around the $z^{\prime}$ axis of the $C S A$, which may be explained by a diffusive rotational motion of the chain segments around the long axis of the molecules ${ }^{8}$. Motional decoupling of chain 


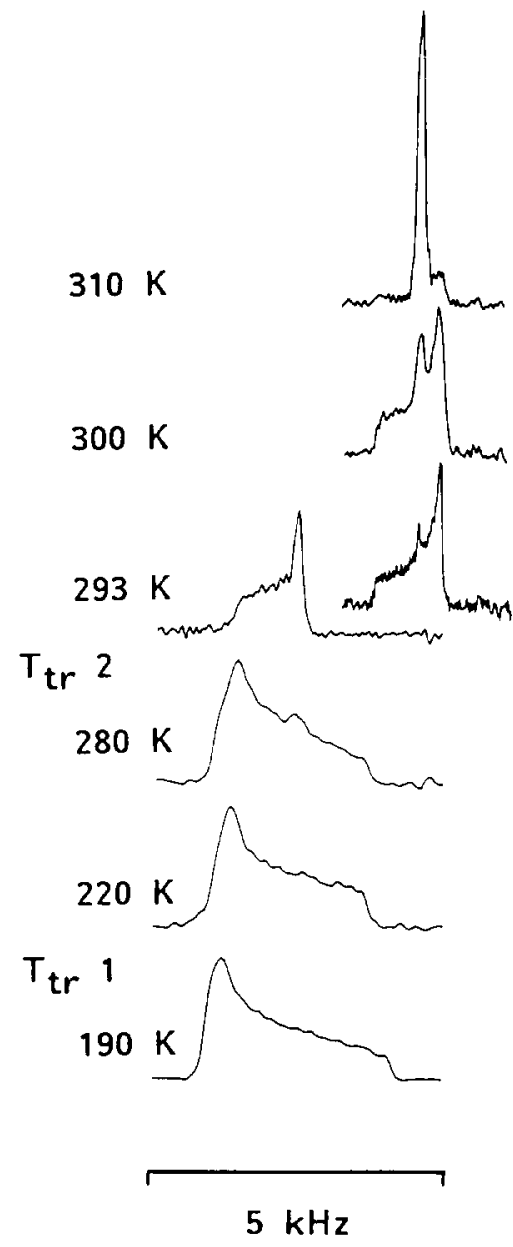

Figure 1 Temperature variation of the ${ }^{29} \mathrm{Si}$ chemical-shift anisotropy of a $\beta$-PDES as recorded with cross-polarization (right column) and by single-pulse excitation without cross-polarization (left column)

segments in an as-yet unknown length is allowed by liquid-like conformational interconversion as indicated by the isotropic chemical shift. Such rotational and translational disordering was already postulated by Papkov, Godovsky et al. from the sharp decrease in the X-ray intensity with $2 \theta^{3,4}$.

Similar $C S A{ }^{29} \mathrm{Si}$ n.m.r. experiments on PDES were performed by Litvinov, Spiess et al. ${ }^{13}$ parallel to this work. They attempted a more quantitative interpretation of the ${ }^{29} \mathrm{Si} C S A$ for $\alpha_{2}$ - and $\beta_{2}$-PDES. Assuming that the $z$-axis of the ${ }^{29} \mathrm{Si} C S A$ is perpendicular to the chain axis, they showed that backbone oscillation with an angle not over $40^{\circ}$ can be responsible for the line narrowing and the deviation from axial symmetry.

The lineshapes of the spectra at $280 \mathrm{~K}$ and at higher temperatures do not represent simple CSA patterns. From comparison of the different spectra, it is obvious that they represent superpositions of the resonances for different PDES modifications. Special care was taken to analyse these superpositions and to separate the effects from non-axial motional narrowing.

The right column in Figure 1 shows ${ }^{29} \mathrm{Si} C S A$ spectra that were recorded by a single $90^{\circ}$ pulse excitation in combination with high-power decoupling and a sufficient delay to ensure complete relaxation of all modifications. The spectrum in the $\mu$-phase clearly consists of the anisotropically averaged $\mu$-phase signal with a width of $1.1 \mathrm{kHz}$ and an isotropically averaged part with a linewidth of about $100 \mathrm{~Hz}$. The fraction of the isotropic portion grows with increasing temperature.

Also the CP spectrum at $280 \mathrm{~K}$ in Figure 1 represents a superposition of different signals. Figure $2 a$ presents the CSA of PDES at $280 \mathrm{~K}$, which was recorded without cross-polarization and a sufficient delay to ensure complete relaxation of all modifications. The sample was rapidly cooled from room temperature to $180 \mathrm{~K}$ and then warmed to $280 \mathrm{~K}$. Thus, it consists of $\beta$ - and $\alpha$-PDES. The main, broad component can be assigned to $\alpha_{2}$ - or $\beta_{2}$-PDES by comparison with spectra recorded at lower temperatures. Also a small isotropically averaged fraction is evident. In contrast, no isotropic component could be observed in cross-polarization experiments.

The components could be separated by means of an inversion recovery experiment ${ }^{15}$, due to the significant difference in ${ }^{29} \mathrm{Si} T_{1}$ relaxation times. A $180^{\circ}$ pulse was followed by a delay time $T_{1}\left(\beta_{2}\right) \geqslant r \geqslant T_{1}(\mu)$. The free induction decay (FID) was recorded after an additional $90^{\circ}$ pulse. The $180^{\circ}$ pulse turned the magnetization into the $-z$ direction. The slowly relaxing component did not change within the delay time, while components with short $T_{1}$ relaxed back into the $+z$ direction. The following $90^{\circ}$ pulse generated an FID that transformed to a negative signal of the slowly relaxing component and a positive signal of the fast relaxing component.

Figure $2 b$ shows a spectrum that was recorded with a delay time of $\tau=25 \mathrm{~s}$. For convenience, the spectrum was inverted by a $180^{\circ}$ phase correction, so that signals that correspond to slow $T_{1}$ times are shown positively. The signal is a superposition of the hardly relaxed broad component, which is observed as a positive signal, and the negative signal of the fully relaxed narrow component. Addition of the two spectra results in the spectrum in Figure $2 c$, which is a one-component non-axially symmetric CSA with $v_{x}-v_{z}=2.6 \mathrm{kHz}$ and $\eta=0.14$. This spectrum represents the $C S A$ of $\alpha_{2}$ - and $\beta_{2}$-PDES without the superimposed narrow component. Figure $2 d$ resulted when the spectrum of Figure $2 b$ was subtracted from that in Figure 2a. The signal consists only of the narrowed component with short relaxation time. Comparison with the spectra above $T_{\mathrm{tr}} 2$ reveals that the spectrum represents a portion of the sample that has already transformed into the $\mu$-phase and, in addition, an isotropic component. The isotropic component indicates an amorphous fraction.

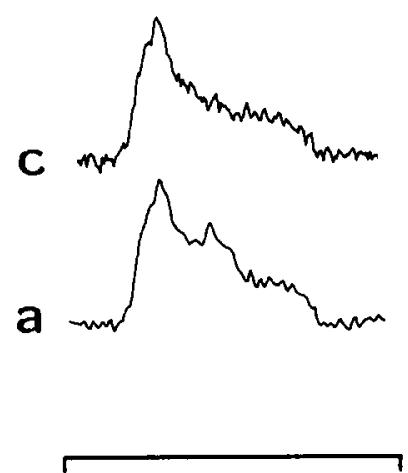

$5 \mathrm{kHz}$

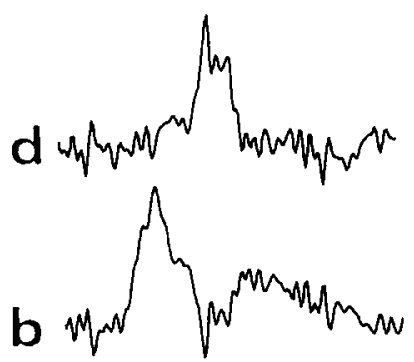

$5 \mathrm{kHz}$
Figure $2{ }^{29} \mathrm{Si} C S A$ of a mixture of $\alpha_{2}$ - and $\beta$-PDES: (a) recorded at $280 \mathrm{~K}$ without cross-polarization; (b) recorded by means of an inversion recovery experiment, $\tau=25 \mathrm{~s}$, the spectrum is $180^{\circ}$ phase corrected; (c) spectrum as obtained by addition of spectra (a) and (b); (d) spectrum as obtained by subtraction of spectrum (b) from spectrum (a) 
The sample was quenched from $300 \mathrm{~K}$, where already a significant part of the sample showed an isotropic mobility in the ${ }^{29} \mathrm{Si} C S A$. In contrast, careful cooling of PDES within the $\mu$-phase resulted in an increase of the crystallinity to almost $100 \%$, as shown by the $293 \mathrm{~K}$ spectrum in Figure 1.

\section{Dielectric relaxation}

The slight narrowing of the ${ }^{29} \mathrm{Si} C S A$ on raising the temperature above the $\alpha_{1}-\alpha_{2}$, respectively $\beta_{1}-\beta_{2}$, transition was explained by the onset of $-\mathrm{Si}-\mathrm{O}-$ librations. Such a reorientation of the $\mathrm{Si}-\mathrm{O}$ segments affects the dielectric properties of PDES ${ }^{16}$ due to the considerable dipole moment of the $\mathrm{Si}-\mathrm{O}$ bond $(q=0.6 \mathrm{D})^{17}$. Figure 3 presents the temperature dependence of the apparent dielectric constant $\varepsilon_{\mathrm{r}}$, which was recorded for $\beta$-PDES at a frequency of $10 \mathrm{kHz}$. No significant frequency dependence was observed between $100 \mathrm{~Hz}$ and $100 \mathrm{kHz}$ at the investigated temperatures. Varying the temperature, an increase of $\varepsilon_{\mathrm{r}}$ was observed at the lower transition. The increase of $\varepsilon_{\mathrm{r}}$ is not accompanied by a significant dielectric loss. Thus, the increase of $\varepsilon_{\mathrm{r}}$ monitors the onset of cooperative oscillations of the backbone at the $\beta_{1}-\beta_{2}$ transition in support of the ${ }^{29} \mathrm{Si}$ n.m.r. experiments. The decrease of $\varepsilon_{\mathrm{r}}$ at the upper transition can be explained by the decrease of the density of the sample $(12 \%)^{4}$ and thus a decrease of the orienting dipoles per volume. No additional increase of the main-chain mobility is observable by dielectric relaxation at the $\beta_{2}-\mu$ transition and at the isotropization temperature.

\section{Microscopy}

On annealing, $\alpha_{2}$-PDES converts into the thermodynamically more stable $\beta_{2}$-PDES. We observed about $50 \%$ conversion within $6 \mathrm{~h}$ at $260 \mathrm{~K}$ for a sample that consisted predominantly of $\alpha_{2}$-PDES. Molecular packings in both crystal modifications are similar, and even become indistinguishable by high-resolution solid-state n.m.r. experiments. In view of the high molecular mobility, which is observed by ${ }^{13} \mathrm{C}$ n.m.r., ${ }^{29} \mathrm{Si}$ n.m.r.,

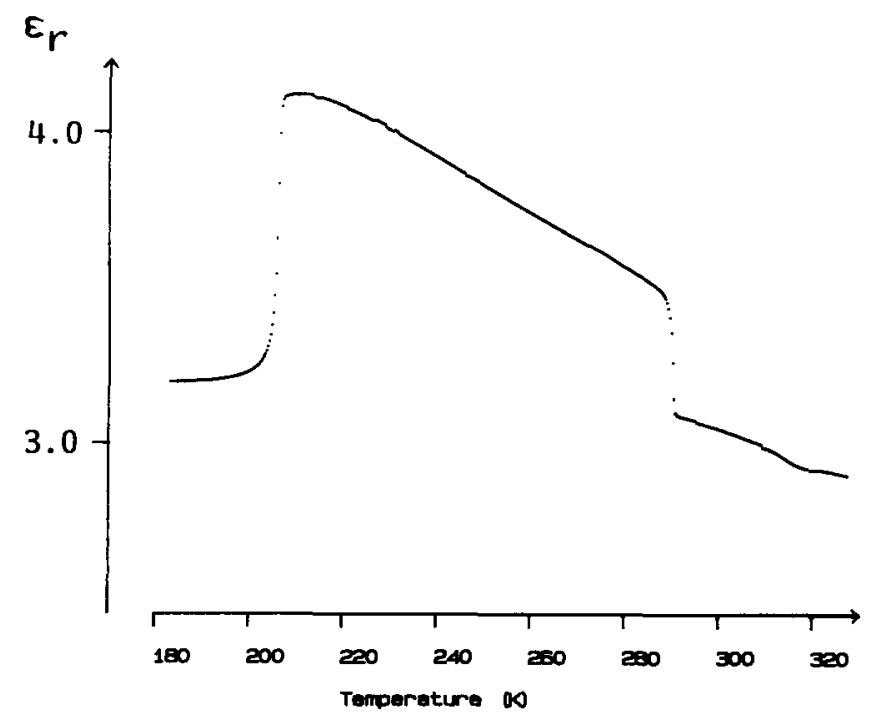

Figure 3 Temperature dependence of the dielectric constant $\varepsilon_{\mathrm{r}}$ of pure $\beta$-PDES dielectric relaxation and also Raman spectroscopy?, rearrangement on small scales should occur almost instantaneously. Thus, the slow conversion of $\alpha_{2}$-PDES into $\beta_{2}$-PDES indicates that long-range molecular motion with long correlation times is necessary to transform $\alpha_{2}$ into $\beta_{2}$-PDES. This implies differences in the crystallite morphology. The question was studied by transmission electron microscopy on replicas of PDES fracture surfaces. The $\alpha_{2}$ and $\beta_{2}$-PDES samples were prepared by the procedures described before, and afterwards cooled rapidly to $-150^{\circ} \mathrm{C}$. Surface replicas were prepared from freshly broken samples by vacuum coating with carbon/platinum. The replicas of the deep-frozen samples represent the crystallite morphologies of the corresponding PDES modification.

Figure 4 shows micrographs that were obtained for $\alpha$ - and $\beta$-PDES. Figure $4 a$ represents a replica of a $\beta$-PDES sample. The orientation of the fracture surface was perpendicular to the steel support on which the sample was crystallized in the careful annealing process necessary to obtain pure $\beta$-PDES. Crystallite layers of $2000-5000 \AA$ enclose a triangle with a flat non-regular structure in the centre of the photograph. This might represent the surface of a lamella whose orientation is parallel to the image plane. The most important point appears to be the thickness of the lamellae, which indicates the formation of extended-chain crystals. Comparison with pictures of chain-folded and anabaric polyethylene samples ${ }^{18,19}$ shows a great similarity to the morphology of extended-chain polyethylene crystals.

Almost unstructured images as shown in Figure $4 b$ resulted when a $\beta$-PDES sample was broken parallel to the support on which the sample was annealed. Probably, only lamellae surfaces are observed. As the specimen had a thickness of more than $1 \mathrm{~mm}$, this demonstrates strong orientation of the crystal axis by the support surface during annealing in the mesomorphic state.

A significantly different morphology was observed for fracture surface replicas of $\alpha$-PDES as shown in Figure $4 c$. Lamellae with a thickness of about 300-600 can be observed. This is in agreement with SAXS data, which showed long periods of corresponding length, and gives evidence for a chain-folded crystal morphology in the case of $\alpha$-PDES.

A replica of a sample that contains both polymorphs is shown in Figure $4 d$. Besides thin lamellae with a thickness of about $500 \AA$ on the left of the picture, large flat crystal faces can be observed, which are up to $8000 \AA$ and are separated by softly bent depressions.

In summary, the electron micrographs demonstrated lamellar crystallization in case of $\alpha$-PDES and extendedchain crystallization in the case of $\beta$-PDES. We could not observe thin lamellae in $\beta$-PDES samples. Extended crystal faces in $\alpha$-PDES samples might be assigned to domains of $\beta$-PDES, as know that the $\alpha$-PDES samples practically always contain some $\beta$-PDES.

In the highly disordered but still anisotropic $\mu$-phase, the liquid-like segment mobility is large enough that the samples can easily flow. Figure $5 a$ presents optical micrographs of PDES under crossed polarizers. As reported also before ${ }^{6}$, the sample is birefringent and distinct crystallites can be observed. Figure $5 b$ presents the micrographs after shearing at $300 \mathrm{~K}$. The distinct crystallites disappeared and the birefringence became uniform, which indicates that the sample was oriented by shearing. 


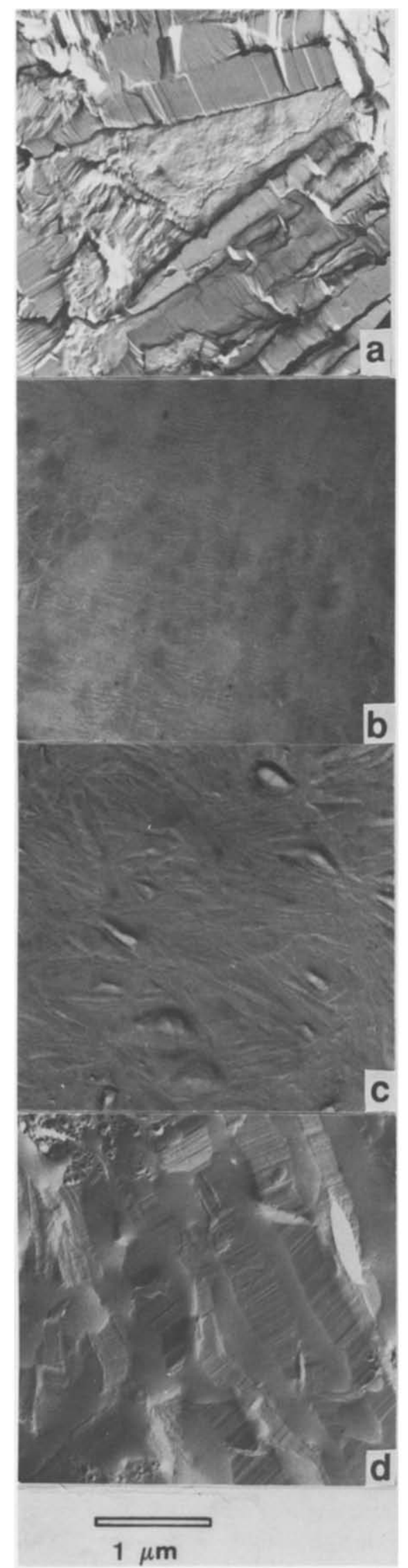

Figure 4 Electron micrographs of a platinum/carbon replica of the fracture surface of $\alpha$ - and $\beta$-PDES: (a) $\beta$-PDES fracture surface perpendicular to the support on which the sample was annealed; (b) $\beta$-PDES fracture surface parallel to the support on which the sample was annealed; (c) $\alpha$-PDES; (d) sample that contains both modifications

\section{Viscoelasticity}

To investigate changes in the viscoelasticity upon the transition from the isotropic state to the $\mu$-phase, we performed simple shear and also creep recovery experiments. A constant stress $(\sigma)$ was applied to shear the sample and the resulting deformation $(\gamma)$ was measured as a function of the time by means of a cone-and-plate fixture. Figure $6 a$ presents a creep recovery experiment at $300 \mathrm{~K}$ in which a stress of $\sigma_{0}=3000 \mathrm{~N} \mathrm{~m}^{-2} \mathrm{~K}^{-1}$ was applied. At this temperature, the sample is in the $\mu$-state. The material showed a viscoelastic behaviour, and the recoverable compliance $J_{\mathrm{e}}(t \rightarrow \infty)=8 \times 10^{-5} \mathrm{~m}^{2} \mathrm{~N}^{-1}$ was (within experimental error) equal to the compliance calculated from the intercept of the steady-state shearing, $J_{\mathrm{e}}(t \rightarrow 0)=6.5 \times 10^{-5} \mathrm{~m}^{2} \mathrm{~N}^{-1}$. An apparent viscosity of $\eta=9 \times 10^{7} \mathrm{~N} \mathrm{~s} \mathrm{~m}^{-2}$ resulted after about $2 \mathrm{~h}$ according to the relation $\eta=\sigma_{0} / \gamma$. (It must be noted that the viscosity is no longer scalar because of the anisotropy in the $\mu$-phase.) Figure $6 b$ represents the same creep recovery experiment with a much longer shearing time. Considerable shear thinning occurred after about $5 \mathrm{~h}$. The apparent viscosity decreased from $6.5 \times 10^{7} \mathrm{~N} \mathrm{~s} \mathrm{~m}^{-2}$ after $2 \mathrm{~h}$ to $1.6 \times 10^{7} \mathrm{Ns} \mathrm{m}^{-2}$; however, the value remained larger than the melt viscosity. Figure 7 represents the temperature dependence of the steady-state viscosity. The viscosity dropped by five orders of magnitude when the temperature was raised above the isotropization transition and the flow behaviour became Newtonian. No shearrate dependence was observed even for the highest applied stress $\left(36000 \mathrm{Nm}^{-2}\right)$. Below the transition to the $\alpha$ - or $\beta$-phase, the viscosity was too high to be determined.

The experiments described show different aspects of the PDES sample. Large-scale deformation of $\mu$-PDES results in a considerable decrease of the apparent viscosity, which can be explained by 'crystallite' alignment of the anisotropic material. The viscosity of $\mu$-PDES is considerably higher than that of the isotropic melt, independently of the shear-rate and orientation effects. The $\mu$-PDES was viscoelastic, although the molecular weight of the investigated sample was too low to observe elastic behaviour of the isotropic melt. The observation of a recoverable compliance and the increase in shear viscosity on the formation of $\mu$-PDES is equivalent to some physical crosslinking. Three deformation processes are indicated: orientation of anisotropic domains, viscous deformation of the oriented sample, and recoverable elastic deformation.

\section{DISCUSSION}

The observed changes of the molecular structure and segmental motions of the different PDES modifications can be correlated with the changes in mechanical properties and the results from thermal analysis.

The two polymorphs can be distinguished clearly in the crystalline state below the lower disordering transition by n.m.r. spectroscopy. The large differences that we observed in the chemical shifts indicate significant differences in the molecular packing and chain conformations. This appears to be in agreement with different crystal structures as observed by X-ray experiments on $\alpha$ - and $\beta$-PDES samples ${ }^{4}$. No mobility of the backbone was observed while the onset of oscillations around the $\mathrm{Si}-\mathrm{C}$ bond is indicated by partial averaging of the ${ }^{13} \mathrm{C}$ chemical shift anisotropy. 

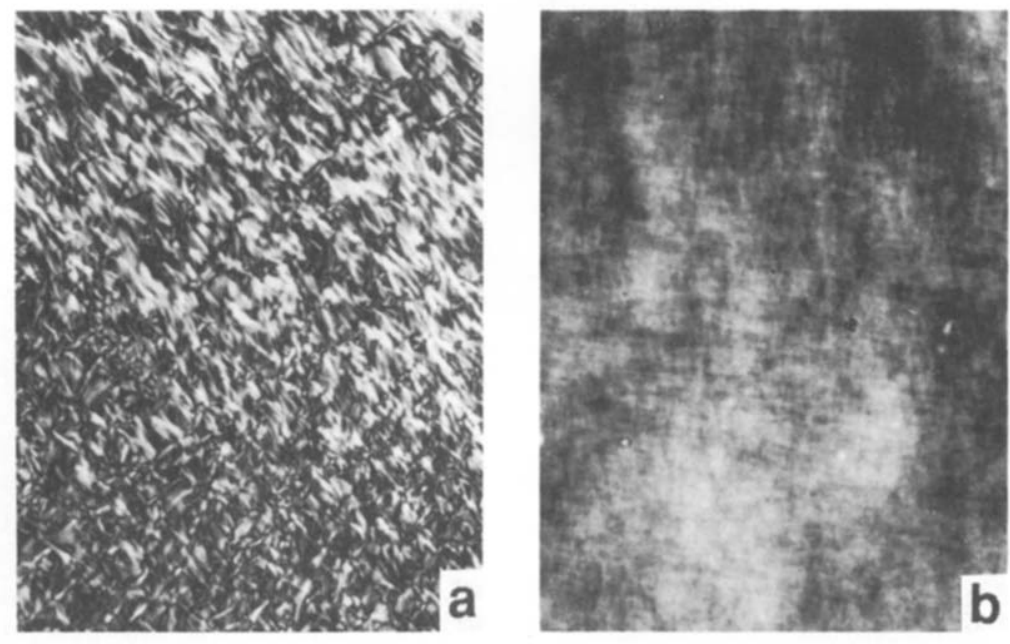

$100 \mu \mathrm{m}$

Figure 5 Optical birefringence of PDES at $300 \mathrm{~K}$ in the $\mu$-phase (a) before and (b) after shearing
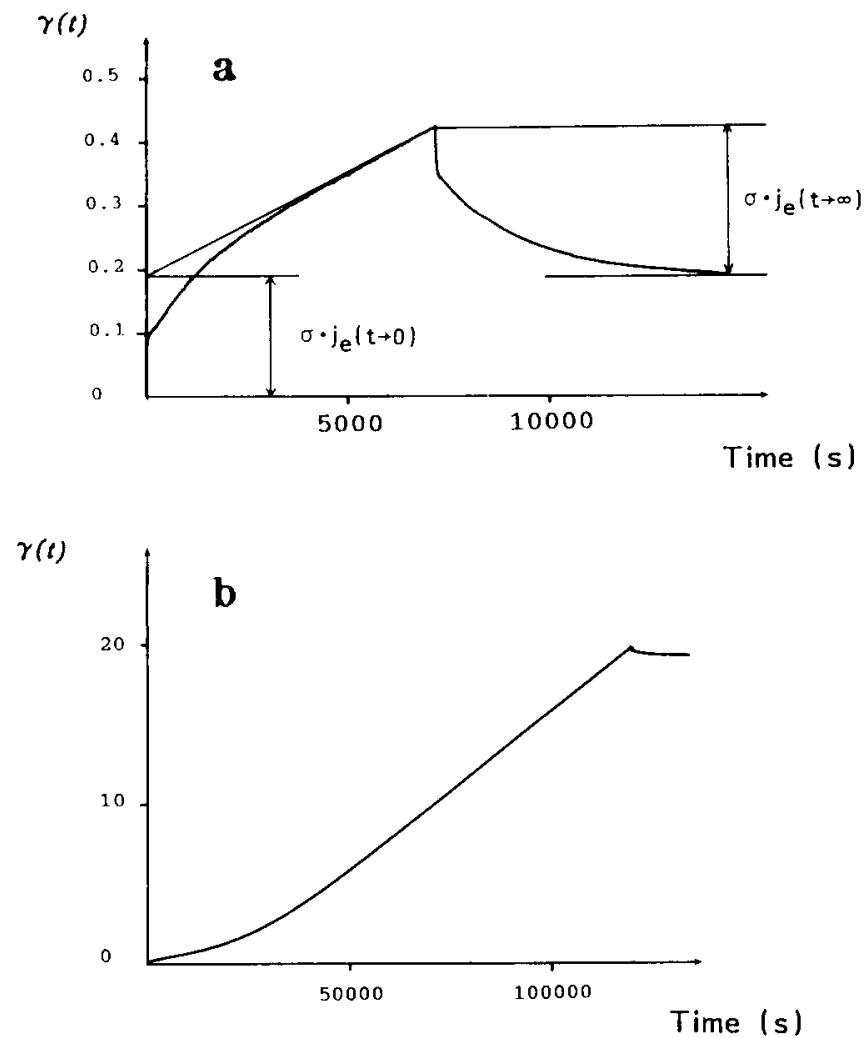

Figure 6 Creep recovery experiment with $\mu$-PDES at $300 \mathrm{~K}$, shear stress $\sigma=30000$ dyn cm$^{-2}$; (a) shearing time $t=14400 \mathrm{~s}$; (b) shearing time $t=134000 \mathrm{~s}$

Significant changes in the molecular conformation of the $-\mathrm{Si}-\mathrm{O}-$ backbone were observed by the n.m.r. experiments at the $\alpha_{1}-\alpha_{2}$ and the $\beta_{1}-\beta_{2}$ transition. In contrast, $\mathrm{X}$-ray experiments on oriented $\alpha$ - and $\beta$-PDES samples showed practically no change in the identity period and X-ray density on the $\alpha_{1}-\alpha_{2}$ and the $\beta_{1}-\beta_{2}$ transition, from which the conclusion on similar segment conformations was drawn ${ }^{4}$. The observed onset of restricted backbone librations is in agreement with

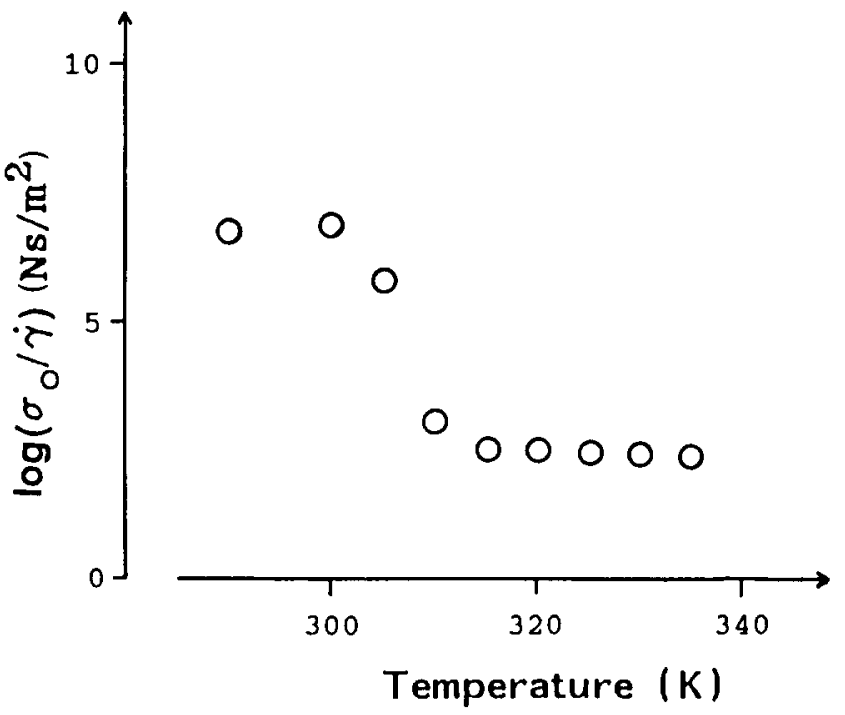

Figure 7 Temperature dependence of the steady-state viscosity $\left(\eta=\sigma_{0} / \gamma\right)$ of PDES

Raman ${ }^{7}$ and ${ }^{1} \mathrm{H}$ n.m.r. ${ }^{11,12}$ data, which have been reported before.

Considerable averaging of the overlapping ${ }^{13} \mathrm{C} C S A$ values and the observed coalescence of the isotropic ${ }^{13} \mathrm{C}$ chemical shifts, which represent the fast exchange average of the $\mathrm{Si}-\mathrm{C}$ bond rotational isomeric states, demonstrate dynamically the disorder of the side-chain conformation. The motional states appear to be similar or even identical for $\alpha$ - and $\beta$-PDES. Although high-resolution ${ }^{29} \mathrm{Si}$ and ${ }^{13} \mathrm{C}$ n.m.r. experiments discriminated $\alpha_{2}$-PDES and $\beta_{2}$-PDES clearly, the difference in the isotropic chemical shifts is small and vanishes with increasing temperature. This indicates that the difference in segmental molecular structure is small and should vanish already below the transition to the $\mu$-phase.

Conversion of $\alpha_{2}$-PDES to the thermodynamically more stable $\beta_{2}$-PDES is unexpectedly slow considering the correlation times of the observed molecular motions 
and the similarity in crystal structures. This could be explained by the morphological differences between the two polymorphs and the necessity for long-range rearrangements to convert chain-folded $\alpha_{2}$-PDES to the thermodynamically more stable chain-extended $\beta_{2}$-PDES. The formation of extended-chain crystals has been discussed also for polyphosphazenes and poly (di-n-alkylsilanes), for which the existence of similar mesophases has been reported ${ }^{20,21}$.

The question arises: why should the segmental packing differ, as observed by $\mathrm{X}$-ray diffraction experiments? In view of the strong interdependences, which we observed for large cyclic alkanes ${ }^{22}$, between the crystal subcell and the adjacent fold structure, chain folding and molecular arrangement in the lamellae surface may influence the molecular packing inside the lamellae to a significant extent. Thus, it appears plausible that the molecular packing inside chain-folded $\alpha_{2}$-PDES lamellae may be influenced by strain originating in the lamella surface, while for a chain-extended $\beta_{2}$-PDES such an effect would not be observable.

An important consequence results from the observation of chain-folded lamellae for $\alpha$-PDES. The $\alpha$-modification is formed when a sample is quenched from the $\mu$-state. Thus, the morphology in the $\mu$-state must be related to the lamellae structure of the $\alpha$-modification.

Dynamic conformational disordering in combination with the preservation of the three-dimensional crystal lattice ${ }^{8}$ characterizes the mesomorphic state, not only $\alpha_{2-}$ and $\beta_{2}$-PDES but also $\mu$-PDES as well. According to ref. 23, these modifications may be classified as variations of 'condis' crystals. Conformational disordering is restricted to the ethyl side-chains in the less disordered $\alpha_{2^{-}}$and $\beta_{2}$-PDES, while in the case of $\mu$-PDES also the inorganic backbone is disordered. It is typical for other condis crystals, like the hexagonal modifications of polyethylene, polytetrafluoroethylene, or trans-1,4-polybutadiene, that they still appear rather solid and that annealing in this mesophase results in nearly $100 \%$ crystallinity. In contrast, creep recovery experiments showed a remarkably fluid character of $\mu$-PDES, and the crystallinity of samples that were annealed in the $\mu$-state was often poor. With respect to its fluidity, $\mu$-PDES resembles the liquid-crystalline (LC) states of rigid-rod polymers. Although PDES does not contain rigid mesogenic units like typical LC polymers ${ }^{24}$, it may be argued that the PDES chains are preferentially elongated due to a repulsive interaction between the organic side-chains and the inorganic backbone, which would explain orientational ordering.

Probably, the coexistence of anisotropic and isotropic motional states of $-\mathrm{OSiEt}_{2}$ - segments, which is demonstrated by the CSA experiments, is an important aspect in the understanding of the properties of $\mu$-PDES. Although the samples are macroscopically homogeneous, we have to consider microscopic inhomogeneities. Consistent with a broad melting range as observed by d.s.c. and $\mathrm{X}$-ray experiments, the proportion of the isotropic fraction increases with temperature. This partial isotropization may be due to the broad molecular-weight distribution and especially to the content of cyclic molecules, which are present due to the chain-ring equilibrium in polysiloxanes. A strong molecular-weight dependence has been reported for the isotropization temperature ${ }^{9,10}$.

Microscopic phase separation within domains of different molecular weights and consequently also different melting points could explain the partial isotropization. In this case, the observed fluid character of $\mu$-PDES could be attributed in part to the liquid, isotropic fraction. So the crystallites might well resemble the more solid-like condis crystals. Partial melting due to molecular-weight inhomogeneity could also explain why we could not prepare samples of $100 \%$ crystallinity on isothermal annealing experiments in the $\mu$-state. An increase in crystallinity could be obtained only by cooling the sample within the mesophase.

The question remains why demixing in such a fluid system should be restricted to small scales. Only a relatively small increase in disorder occurs on isotropization, and also the densities of the $\mu$-state and the isotropic melt do not differ considerably. Owing to the similarity in the free energy and the dynamic nature of both states, we have to consider substantial fluctuation of molecules between the $\mu$-state and the isotropic state, which might help to stabilize the spatial coexistence of the two states. To understand these phenomena better, it appears of great importance to perform similar experiments on samples that are of uniform molecular weight and do not contain cyclic molecules.

Recent experiments, which we shall report in a forthcoming paper, showed the same coexistence of isotropic and anisotropic fractions for poly(di-n-propylsiloxane) and also for poly(di-n-butylsiloxane). Thus, the poly(di$\mathrm{n}$-alkylsiloxanes) represent a class of materials with novel anisotropic properties. The molecular-weight dependence of the width and the temperature of the isotropization transition in connection with the reported effects on the viscosity offers intriguing possibilities to design materials with particular temperature and shear-rate dependences of their viscoelastic properties.

While this paper was in preparation we got notice of molecular mechanics calculations on PDES ${ }^{25}$, whose results agree nicely with the data and interpretation reported above. In summary, because of the steric hindrance of the ethyl group rotation, cooperative side motion dominates the molecular dynamics in the conformational disordered states and contributes to an apparent chain stiffness.

\section{ACKNOWLEDGEMENTS}

The author gratefully acknowledge valuable support by Dr H. Drotloff (synthesis), M. Drechsler and M. Kunz (TEM), H. Seiberle (dielectric experiments), and D. Oelfin and A. Hasendindl (n.m.r.). Financial support was granted by the Deutsche Forschungsgemeinschaft within the SFB 60, Teilprojekt F-5. K. Loufakis thanks the Alexander von Humboldt-Stiftung for a fellowship.

\section{REFERENCES}

1 Lee, C. L., Johannson, O. K., Flanigam, O. L. and Hahn, P. Am. Chem. Soc. Polym. Prepr. 1969, 10 (2), 1319

2 Froix, M. F., Beatty, C. L., Pochan, J. M. and Hinman, D. D. J. Polym. Sci., Polym. Phys. Edn. 1975, 13, 1269

3 Godovsky, Yu. K. and Papkov, V. S. Adv. Polym. Sci. 1989, 88, 1

4 Tsvankin, D. Ya., Papkov, V. S., Zhukov, V. P., Godovsky, Yu. K., Svistunov, V. S. and Zhadanov, A. A. J. Polym. Sci., Polym. Chem. Edn. 1985, 23, 1043

5 Godovsky, Y. K. and Papkov, V. S. Makromol. Chem., Macromol. Symp. 1986, 4, 71 
Papkov, V. S., Swistunov, W. S., Godovsky, Y. K. and Zadanov, A. A. J. Polym. Sci., Polym. Chem. Edn. 1987, 25, 1859

Friedrich, J. and Rabolt, J. F. Macromolecules 1987, 20, 1975

Kögler, G., Hasenhindl, A. and Möller, M. Macromolecules $1989,22,4190$

9 Godovsky, Y. K., Makarova, N. N., Papkov, V. S. and Kuzim, N. N. Makromol. Chem., Rapid Commun. 1985, 6, 443

10 Godovsky, Y. K., Mamaeva, I. I., Makarova, N. N., Papkov, V.S. and Kuzim, N. N. Makromol. Chem., Rapid Commun. 1985, 6, 797

11 Litvinov, V. M., Lavrukhin, B. D., Papkov, V. S. and Zhdanov, A. Polym. Sci. USSR 1985, 27, 1715 (Vysokomol. Soed. (A) 1985. 27, 1529)

12 Litvinov, V. M., Lavrukhin, B. D., Papkov, V. S. and Zhdanov, A. Dokl. Akad. Nauk SSSR 1983, 271, 900

13 Litvinov, V. M., Whittacker, A. K., Hagemeyer, A. and Spiess, H. W. Colloid Polym. Sci. 1989, 267, 681

14 Pines, A., Gilby, M. G. and Waugh, J. S. J. Chem. Phys. 1973, 59,569

15 Cory, D. G. and Ritchey, W. M. Macromolecules 1989, 22, 1611
Beatty, C. and Karasz, F. E. J. Polym. Sci., Polym. Phys. Edn. 1975, 13, 977

17 Mark, J. E. J. Chem. Phys. 1968, 49, 1398

18 Bassett, D. C. 'Principles of Polymer Morphology', Cambr. Solid State Sci. Ser., Cambridge University Press, Cambridge, 1981, Ch. 7

19 Geil, P. H., Anderson, F. R., Wunderlich, B. and Arakawa, T. J. Polym. Sci., Polym. Phys. Edn. (A) 1964, 2, 3707

20 Sun, D. C. and Magill, J. H. Polymer 1987, 28, 1243

21 Miller, R. D. Chem. Rev. 1989, 1359

22 Möller, M., Waldron, R. F., Drotloff, H. and Kögler, G. in 'Integration of Fundamental Polymer Science and Technology', (Eds. P. J. Lemstra and L. A. Kleintjens), Elsevier Applied Science, New York, 1988, Vol. 2

23 Wunderlich, B., Möller, M., Grebowicz, J. and Baur, H. Adv. Polym. Sci. 1988, 87, 1

24 'Liquid Crystalline Order in Polymers', (Ed. A. Blumstein), Academic Press, New York, 1978

25 Miller, K. J., Grebowicz, J., Wesson, J. P. and Wunderlich, B. Macromolecules 1990, 23, 849 